\title{
Saint-André-de-Rosans - Prieuré : église prieurale et cellier
}

\author{
Marie-Pierre Estienne
}

\section{OpenEdition \\ Journals}

Édition électronique

URL : http://journals.openedition.org/adlfi/6710

ISSN : 2114-0502

Éditeur

Ministère de la culture

Référence électronique

Marie-Pierre Estienne, « Saint-André-de-Rosans - Prieuré : église prieurale et cellier », ADLFI.

Archéologie de la France - Informations [En ligne], Provence-Alpes-Côte d'Azur, mis en ligne le 01 mars

2007, consulté le 01 mai 2019. URL : http://journals.openedition.org/adlfi/6710

Ce document a été généré automatiquement le 1 mai 2019.

(c) Ministère de la Culture et de la Communication, CNRS 


\title{
Saint-André-de-Rosans - Prieuré : église prieurale et cellier
}

\author{
Marie-Pierre Estienne
}

\section{Identifiant de l'opération archéologique : 8520}

Date de l'opération : 2007 (SU)

1 Dans le cadre de la restauration du prieuré de Saint-André-de-Rosans, classé au titre des Monuments historiques, et de la présentation dans l'aile occidentale des pavements de mosaïques découverts en 1986-1987 dans l'église prieurale, une surveillance archéologique avait été prévue parallèlement à l'enlèvement de remblais archéologiques situés contre le mur septentrional de l'église (voir NIL PACA 3, 1986, 34-37 ; 4, 1987, 41-42 ; BSR PACA 1992, 58-59 ; 1993, 32-34).

2 Suite à un décaissement intempestif à la pelle mécanique, opéré sans surveillance contre le mur nord de l'église prieurale, une réunion s'est tenue sur place en octobre 2007 afin d'organiser une opération de sauvetage (convention entre l'État, le Conseil général des Hautes-Alpes et l'APA) qui a suivi une double orientation (Fig. ${ }^{\circ} 1$ : Relevé de l'architecte en chef M. Naviglio, avec localisation et zone d'intervention).

3 La préoccupation première a été de rassembler les données liées au décaissement dans la zone Prieuré nord et d'effectuer :

4 - les relevés d'une partie du mur nord de l'église prieurale, mis au jour par le décaissement ;

5 - un relevé et nettoyage de la coupe ainsi constituée par l'arrêt du décaissement en extérieur nord au niveau du troisième pilier $\left(\mathrm{XII}^{\mathrm{e}} \mathrm{s}\right.$.; interne, entre troisième et quatrième travée) ;

6 - une prospection dans les remblais déversés chez un privé et l'organisation du rapatriement des moellons, pierres de taille et autre mobilier archéologique. 
7 Le deuxième pôle d'intervention a touché l'extrémité sud de l'aile occidentale (cellier, cave cuisine) :

8 - surveillance du creusement d'une tranchée effectuée par l'entreprise pour pouvoir introduire verticalement les morceaux de pavements restaurés dans la salle prévue ;

9 - une stratigraphie a pu être relevée au contact et de part et d'autre du mur édifié après la ruine partielle de la partie sud de l'aile occidentale.

10 Nous en avons profité pour orienter l'aménagement muséographique des pièces lapidaires et participer à leur rangement dans la réserve, située également dans le cellier.

\section{Mur nord de l'église prieurale}

11 - Le mur nord externe, ainsi mis à nu sur plus de 2,50 $\mathrm{m}$ d'élévation au niveau de la première et deuxième travée jusqu'à l'angle nord-ouest de la façade occidentale, révèle la présence d'un parement de petits moellons bien assisés, construit dans la tradition du $\mathrm{XI}^{\mathrm{e}}$ s. (Fig. n²: Relevé du mur nord de l'église prieurale). On note un élément particulièrement intéressant : la base et l'arrachement d'un pilastre engagé jouant le rôle de contrefort, destiné à recevoir l'arcade en saillie externe, élément architectonique jadis relevé par G. Barruol sur l'élévation sud de l'église prieurale côté cloître et sur la façade ouest, lecture désormais effacée par la restauration des Monuments historiques (Société d'études des Hautes-Alpes 1989, p. 125 et fig. 22 et 24, p. 133, fig. 30).

12 Leur édification s'inscrivait dans le projet de voûtement (fin $\mathrm{XI}^{\mathrm{e}} \mathrm{s}$.) de l'église prieurale charpenté, projet avorté avant la construction au XII ${ }^{e}$ s. de la voûte en berceau semibrisé : «Les contreforts extérieurs ont bien été construits au sud, reliés par des arcs en plein-cintre ; arcs et contreforts ont disparu mais leurs arrachements sont bien visibles » notent Y. Esquieu et J. Ulysse.

13 - La berme artificielle du remblai décaissé dessine une coupe nord-sud contre le mur nord, au niveau du deuxième pilier interne duXII ${ }^{e}$ s. Le nettoyage sur une bande de 0,50 $\mathrm{m}$ de large, au pied de la berme laissée à la fin du décaissement, a mis au jour un niveau de pierres plates bien disposées à plat sur un niveau non fouillé. Ces dernières présentent des traces de surchauffe et correspondent à un niveau de sol, type pavement de carreaux taillés dans le grès, qui a subi un incendie. Bien qu'étrangement situées dans un espace jusque là interprété comme extérieur à l'église, elles sont très semblables à celles retrouvées dans la deuxième travée et fouillées dans la partie méridionale de la nef.

14 Il faudra dans de prochaines études replacer l'interprétation de ce sol dans la logique de la circulation et de l'évolution de cet ensemble prieural bénédictin.

15 L'étude de ce niveau de sol exigera vigilance et analyse lors de toute intervention à venir, comme celle du niveau qui le gèle : un remblai de $0,80 \mathrm{~m}$ d'épaisseur, révélant en coupe la présence d'ossements humains, de fragments de tegulae et de verre très fin, d'un tesson de céramique de commune grise. L'ensemble était recouvert par plusieurs remblais (destruction, stockage de pierres par les Monuments historiques et niveau de chantier de restauration en 1990). 


\section{L'aile occidentale}

16 brisée, abritait cave, cellier et cuisine du prieuré ; elle fut reconvertie en mairie et école à
la Révolution, avant la construction de l'actuelle mairie-école édifiée en 1883. Dès lors, cette aile abrite le four communal toujours conservé. L'arrachement de voûte rappelle que l'espace aujourd'hui en extérieur correspondait à un espace intérieur, le mur sud médiéval s'alignant plus au sud, sur le mur méridional du réfectoire (voir textes de 1511). d'utilisation antérieur à la reconversion des lieux et sont sans doute à rattacher - au vu du matériel trouvé - au dernier temps d'occupation de la salle en tant que cellier médiéval : $25 \mathrm{~cm} \times 8 \mathrm{~cm} \times 15 \mathrm{~cm}$ ) comprend deux tessons de céramique vernissée (glaçure jaune interne sur engobe), à paroi épaisse. Elle se trouvait accolée au mur à $0,46 \mathrm{~m}$ sous la pierre du seuil (XVII ${ }^{e}$ s. et $\mathrm{XVIII}^{\mathrm{e}}$ s.) ;

22 - une calade composée de petites pierres calcaire (US 012), noyées dans une terre noire (US 011), installée au-dessus devant la porte d'accès ;

23 - l'ensemble était recouvert d'une terre rapportée par le piétinement et le ravinement par l'eau de pluie (US 010).

24 À l'intérieur, le niveau de sol de l'aile ouest en carreaux de terre cuite correspond à la dernière utilisation de cette aile reconvertie; il repose sur un remblai de tout-venant comprenant des matériaux de construction : tuiles, imbrices, fragments de carreaux, mortier.

25 Cette opération souligne l'importance des dégâts causés par un décaissement sans surveillance archéologique dans un monument classé ; elle souligne le potentiel encore important susceptible d'être révélé par ce monument, bien au-delà d'une « simple » étude de bâti.

ESTIENNE Marie-Pierre

\section{ANNEXES}


Fig. $\mathrm{n}^{\circ} 1$ : Relevé de l'architecte en chef M. Naviglio, avec localisation et zone d'intervention

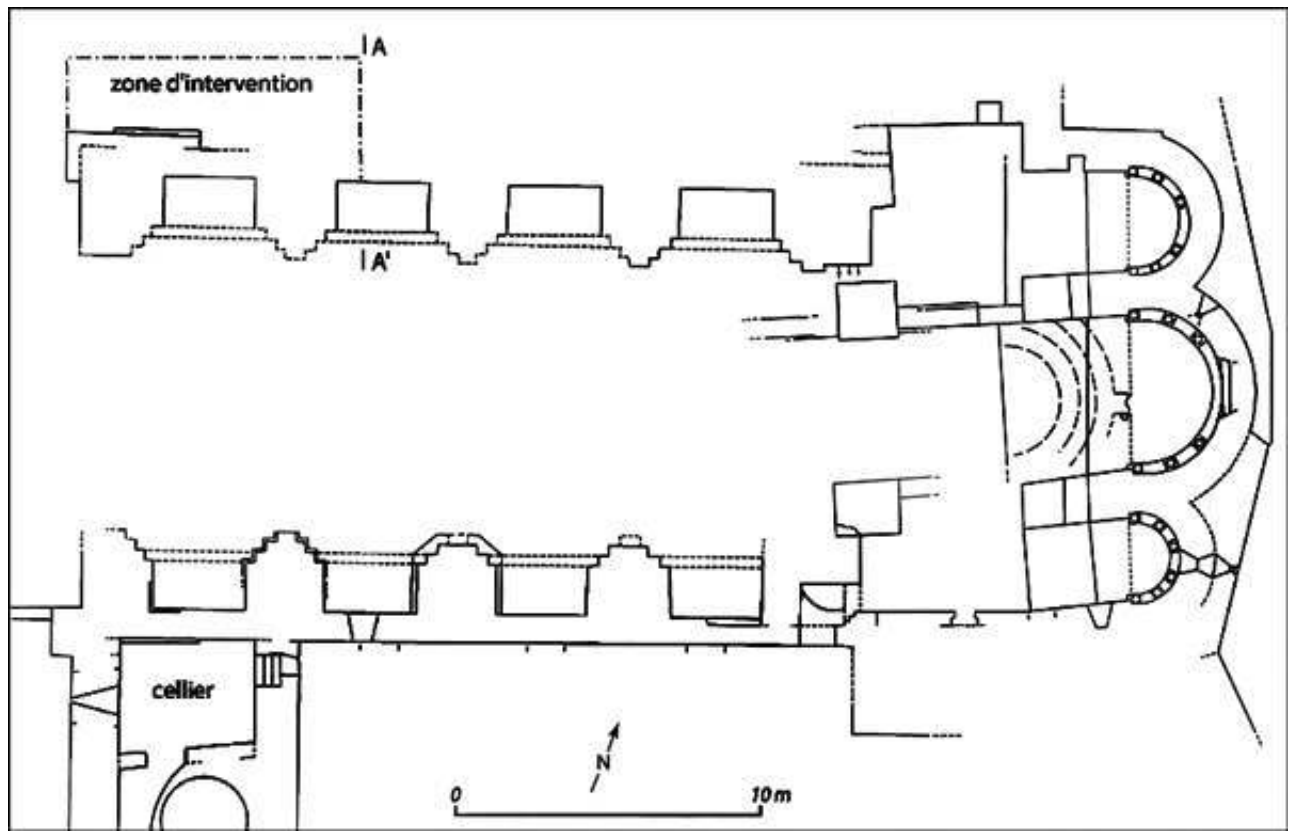

Auteur(s) : Naviglio, M. Crédits : ADLFI (2007)

Fig. $n^{\circ} 2$ : Relevé du mur nord de l'église prieurale

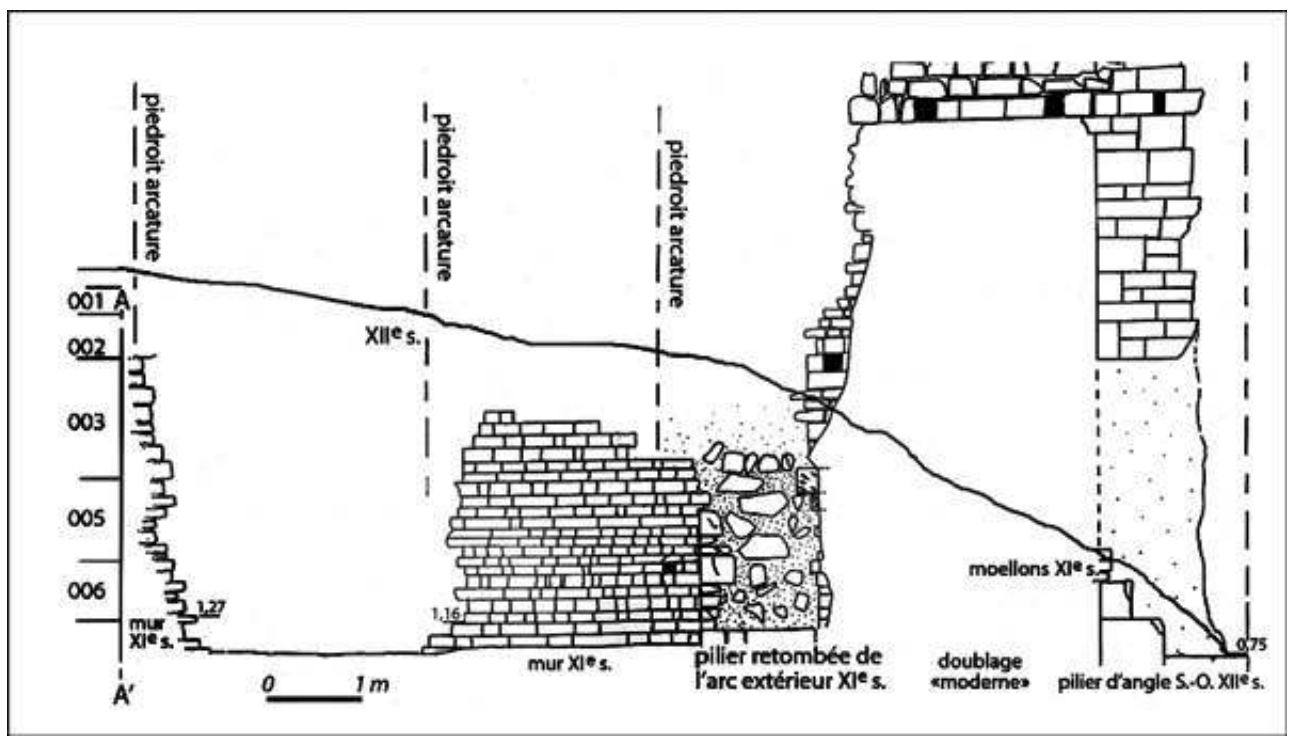

Auteur(s) : Estienne, Marie-Pierre. Crédits : ADLFI (2007)

INDEX

Index chronologique : Moyen Âge, Temps Modernes, XIe siècle apr. J.-C., XIIe siècle apr. J.-C., XVIIe siècle apr. J.-C., XVIIIe siècle apr. J.-C.

Index géographique : Provence-Alpes-Côte d'Azur, Hautes-Alpes (05), Saint-André-de-Rosans operation Sauvetage urgent (SU) 
AUTEURS

MARIE-PIERRE ESTIENNE

AUT 